Something in the way she moves: biological motion, body shape, and attractiveness in women

Edward R Morrison, Hannah Bain, Louise Pattison, Hannah Whyte-Smith

Centre for Comparative and Evolutionary Psychology, University of Portsmouth, Portsmouth, UK

Department of Psychology, University of Portsmouth, King Henry 1 Street, Portsmouth, PO1 2DY, UK. Correspondence to: ed.morrison@port.ac.uk

Funding details: This work received no funding.

Disclosure of interest: The authors report no conflict of interest. 


\title{
Something in the way she moves: biological motion, body shape, and attractiveness in women
}

\author{
Most previous research into the attractiveness of women's bodies has relied on \\ static stimuli such as line drawings or photographs, particularly focusing on the \\ role of body-mass index (BMI) and waist-to-hip ratio (WHR). However, real \\ attractiveness judgments are invariably made on moving bodies, and movement \\ may contain important information about attractiveness. We measured the \\ importance of movement in attractiveness judgments by using motion-capture to \\ isolate dynamic cues from 37 female walkers, and compare ratings of 75 \\ participants made on these, static photographs, and the original videos. Multiple \\ regression analysis revealed that both dynamic and static cues were important in \\ the attractiveness of women's bodies. Furthermore, BMI and WHR predicted \\ attractiveness, but BMI was more important in dynamic rather than static cues, \\ while WHR was important for both static and dynamic cues. These findings \\ suggest that movement is plays a crucial part in the attractiveness of female \\ bodies and cannot be ignored in studies of human mate choice. Furthermore, \\ dynamic and static cues may contain differential information related to female \\ body shape, which further research should attempt to elucidate.
}

Keywords: motion-capture; attractiveness; body shape; biological motion

\section{Introduction}

Like all animals, human mate preferences can be considered in terms of sexual selection. This theory predicts that what people find attractive is that which enhances fitness. Research on the attractiveness of female bodies has focused on two attractiveness cues: waist-to-hip ratio (WHR, the circumference of the waist relative to that of the hips) and body-mass index (BMI, a measure of weight scaled for height). Some research suggests that a low WHR of around 0.7 is most attractive (e.g., Singh, 1993). A low ratio may represent a distribution of fat that is associated with high fertility (Zaadstra et al. 1995; 
Wass, Waldenström, Rössner, \& Hellberg, 1997), and fat reserves in the mothers' hips have been suggested to influence fetal brain development (Lassek \& Gaulin, 2007), making WHR a putative marker of reproductive value.

A problem with such research is that by manipulating the WHR of twodimensional figures, BMI also changes. In fact, multivariate comparison of WHR and BMI suggests that BMI is a much stronger predictor of attractiveness than WHR (e.g Tovée, Maisey, Emery, \& Cornelissen, 1999). For a review of this debate, see Cornelissen, Tovée and Bateson (2009). Another issue with WHR research using drawings of photographs is that there is a difference between the width of the hips relative to the waist, which is what can be perceived from a photograph, and the circumference of the waist relative to the hips, which cannot be seen in a two-dimensional image and must be measured in real life (Tovée, Maisey, Emery, \& Cornelissen, 1999). Despite over two decades of research, the debate still rages over the determinants of attractive female body shape.

Most of the research reviewed above has relied on static images, either linedrawings or photographs. These stimuli may lack ecological validity as face-to-face attractiveness judgments are invariably of 3-dimensional, dynamic bodies. Moving stimuli may be superior for two reasons. First, they provide more information about the shape of the body by presenting static cues from several angles, allowing the viewer to build a more accurate 3-dimensional representation of their target (Bloj, Kersten \& Hurlbert, 1999; Knappmeyer, Thornton, Etcoff, \& Bülthoff, 2002; Ullman 1979). Indeed, Rilling and colleagues (2009) used rotating 3-dimensional body stimuli to show that a novel anthropometric measure - abdominal depth - was more important than BMI or WHR in female attractiveness. However, this study deliberately used a restricted BMI range of 18-24, which may well have limited the explanatory power of this variable 
compared with real life judgements. Second, the movement itself may contain important cues about attractiveness.

Body movement has been well studied since Johansson (1973) showed how a point-light display of a body is meaningless when static, but looks strikingly human when in motion. Point-light techniques have been used to show that body movement can provide information about age, (e.g., Montepare \& Zebrowitz-McArthur, 1988), identity (e.g., Cutting \& Kozlowski, 1977), specific actions people are performing (e.g., Dittrich, 1993), sex (e.g., Barclay et al., 1978), and even sexual orientation (Ambady, Hallahan, \& Conner, 1999). ). In terms of trait judgements dynamic cues are important in judgements of health (e.g. Kramer, Arend \& Ward, 2010), and perceived personality and attractiveness (Thoresen, Vuong \& Atkinson, 2012).

Motion-capture is the modern descendant of the point-light technique and it allows dynamic information to be isolated from static information. Motion-capture studies of human gait have shown that women prefer masculine movement, especially when they are in the fertile phase of their menstrual cycles and when they are more open to short-term mating (Provost, Troje \& Quinsey, 2008). These findings parallel work on facial motion, which suggests that sex-typical movement is attractive in female faces (Morrison, Gralewski, Campbell, \& Penton-Voak, 2007), and that women’s preferences shift across the menstrual cycle (Morrison, Clark, Gralewski, Campbell, \& Penton-Voak, 2009). Women's gait itself changes across the menstrual cycle, but, surprisingly, men prefer the gait of women in the luteal, non-fertile phase (Provost, Quinsey, \& Troje, 2008). In a study using degraded videos rather than motion capture, attractive dances were associated with low digit ratio, a putative marker of high prenatal testosterone exposure and biological quality (Fink, Seydel, Manning, \& Kappeler, 2007). 
Movement is also important in the mating behaviour of many species. For example, female fruitflies choose males on the basis of their ability to dance, which signals their neuromuscular condition (Maynard-Smith, 1956). Likewise, male funnelweb spiders that sway their abdomens at high frequency are more successful at mating (Singer et al., 2000). Video editing and computer animation techniques have allowed experimental manipulation of such dynamic behaviours while keeping other cues constant. For example, video footage of male sticklebacks edited to manipulate the tempo of their courtship displays revealed that females prefer sequences that were speeded up (Rowland, 1995).

While female body shape and gait have been studied independently with respect to sexual selection, they have not been linked. By comparing the attractiveness ratings given to pictures (containing only static cues), point-light walkers (containing only dynamic cues), and videos (containing static and dynamic cues), we can also estimate the relative importance of movement in overall female bodily attractiveness. We also aimed to investigate the association between WHR, BMI, and the attractiveness of static, dynamic, and combined cues. If the relative importance of these two variables differs depending on the stimulus being viewed, it would suggest that static and dynamic cues emphasize different aspects of body shape.

\section{Methods}

\section{Filming}

Thirty-seven female participants (hereafter "models”) were selected from a volunteer sample of university students. The models gave written informed consent, agreeing to being filmed and subsequently rated for attractiveness. The models were given standardized clothing to wear-blue leggings and a grey vest top-which was close fitting so the shape of their body could still be seen. A full body photograph was then 
taken against a white wall using a Nikon D90 18-105 camera. Height was measured with a stadiometer, weight with scales, and waist and hip circumference with a tape measure. Two measurements were taken to ensure reliability, and if the readings were not identical a third was taken to confirm.

Motion capture was achieved with an eight-camera (500 Hz, full resolution) Oqus automated tracking system incorporating two high speed (1000 Hz, full resolution) cameras (Qualysis, Sweden). This equipment picks up reflections from reflective markers positioned on the body. These were calibrated to allow optimal filming where each marker could be seen by at least two cameras to accurately record the three-dimensional position. Reflective markers were attached outside the clothes on the outside of the ankles, knees, hips, shoulders, elbows and wrists, in the middle of their forehead, inbetween their clavicles and on the navel. The models were instructed to walk on a treadmill, which was set to a comfortable walking pace of $2.5 \mathrm{~km}$ per hour, while they were filmed by a Panasonic NV-GS17 camcorder on a tripod at a fixed distance (figure 1, and see supplementary materials for example movies) and the motion-capture cameras. The models walked for about 30 seconds. The point-light videos were edited using Windows Movie Maker to 10-second clips in which the quality of filming was optimal (i.e. markers were not occluded and "lost" by the cameras).

Using Adobe Premier Pro, faces were cut out of the videos so only the body was visible (see supplementary materials). The static images were edited using iPhoto. To obtain the body images (without head), the full-length images were cropped from just below the chin to just below the ankles (figure 1).

\section{Rating}

Seventy-five participants with an average age of 19.6 years (SD 3.1) rated the stimuli for attractiveness. Twenty-five viewed the videos (15 female), 25 viewed the pictures (14 
female), and 25 viewed the point-light walkers (14 female). There were no significant differences between male and female ratings so they were combined. Because independent groups rated each set of stimuli, no participant saw the same model twice, avoiding the possibility of carry-over effects. Stimuli were presented in randomized order on a computer. Ratings were made on a Likert scale of 1 (very unattractive) to 7 (very attractive). All participants gave written informed consent before starting. Videos were presented for 5 seconds, point-light walkers for 10 seconds. Participants were either psychology undergraduates who completed the study in return for course credit, or students at a local college who did the experiments as part of a "psychology day” where they had the opportunity to participate in several unrelated experiments. The study was approved by the psychology ethics committee of the institution where the research was conducted.

\section{Results}

Cronbach's alpha was calculated for each set of stimuli to assess reliability. Reliability was acceptable for the point-light videos (“dynamic attractiveness”, $\alpha=.95$ ), pictures (“static attractiveness", $\alpha=.75$ ), and videos ("static+dynamic attractiveness", $\alpha=.82$ ). The data were therefore averaged across participants for a by items analysis where the stimulus was the statistical unit of analysis. Previous research has suggested that BMI has a curvilinear relationship with attractiveness (e.g. Tovée, Maisey, Emery \& Cornelissen, 1999). Therefore we compared quadratic and linear models for BMI before using it in further analyses. For the motion-capture walkers, the linear model explained the same amount of variance as the quadratic $\left(r^{2}=.30\right)$, so it was treated as a linear variable. Quadratic models explained more variance than the linear models for the videos $\left(\mathrm{r}^{2}=.19\right.$ vs .14), and the pictures $\left(r^{2}=.15\right.$ vs .02$)$. We therefore calculated the vertices of these two quadratic curves to find the most attractive BMI, which were 19.75 for the videos 
and 22.92 for the pictures. These values represent the peak of attractiveness. Lower values for thinner bodies or higher values for fatter bodies were less attractive. WHR, on the other hand, had a linear relationship - the smaller the more attractive. To make both variables comparable on a linear scale we then subtracted each model's BMI from this optimum value and took the absolute difference to estimate their deviation from the most attractive value $\left(\mathrm{BMI}_{\text {dev_static+dynamic }}\right.$ and $\left.\mathrm{BMI}_{\text {dev_static }}\right)$. These values were used in subsequent analyses. All data were normally distributed so parametric statistics were used (table 1). Mean attractiveness for pictures was 4.0 (SD 0.62), for point-light walkers 4.0 (SD 0.88), and for the videos 4.1 (SD 0.87). Figure 2 shows the distribution of body shapes of the models.

\section{Static and dynamic cues}

The correlation between attractiveness of the same woman from static and dynamic cues was quite high, $\mathrm{r}(37)=.61, \mathrm{p}<.01$, suggesting that people who were attractive in a photograph were also attractive in a video. The correlation between attractiveness of the same woman's static+dynamic and dynamic cues was high, $\mathrm{r}(37)=.73, \mathrm{p}<.01$, suggesting that people whose gait was attractive were also attractive in a video. To explore the role dynamic and static cues in overall attractiveness, a regression model was run with static+dynamic attractiveness as the dependent variable, and attractiveness of the static and dynamic cues as independent variables. The overall model was significant, accounting for $63.1 \%$ of the variance, $F(2,34)=23.32, \mathrm{p}<.01$. Attractiveness of both static and dynamic cues predicted static+dynamic attractiveness. We tested for an interaction term (the product of the variables centered around their means), but it was not significant, suggesting that static and dynamic are independent cues of attractiveness, and so it was dropped from the model (table 2).

\section{Body shape and attractiveness}


BMI (or BMIdev_static+dynamic and BMI $I_{\text {dev_static) and }}$ WHR were entered as independent variables in 3 regression models, with static attractiveness, dynamic attractiveness, and static+dynamic attractiveness as dependent variables. Interaction terms were also included, but these were not significant and so were dropped from the final models. For static attractiveness, the overall model was significant, $F(2,34)=6.4$, $\mathrm{p}<.01$, explaining $29.4 \%$ of the variance (adjusted R squared). WHR was a significant predictor, while BMI was not. For dynamic attractiveness, the overall model was significant, accounting for $34 \%$ of the variance, $F(2,34)=10.4, \mathrm{p}<.01$. Both BMI and WHR were significant predictors, but BMI had a larger $\beta$ value. For static + dynamic attractiveness, the overall model was significant, accounting for $38 \%$ of the variance, $F(2$, $34)=11.3, \mathrm{p}<.01$. Both BMI and WHR were significant predictors, with similar $\beta$ values (table 3). Post-hoc power analysis showed that a sample size of 37 offered $93 \%$ power for the lowest R-squared value of $29 \%$ with two predictors (alpha $=0.05$ ).

\section{Discussion}

This study is the first to compare the relative importance of static cues, which can be seen in a photograph, and dynamic cues, which are isolated using motion-capture, in judgments of attractiveness made on women's bodies. Our results show that both dynamic and static cues are important. This is surprising, because most research has focussed on static cues, perhaps because they are easier to study, but also perhaps because our intuitions suggest they accurately index overall attractiveness. Indeed, this intuition is not misleading, because the correlation between attractiveness in a photograph and video was high. However the correlation between attractiveness of gait and the video was similarly high, clearly suggesting the importance of movement in female bodily attractiveness. Our findings add to the body of research that dynamic cues are important in judgements of 
attractiveness (Fink, Weege, Neave, Pham \& Shackeldord, 2015; Thoresen et al. 2012) and should receive as much attention as static cues do.

Our results also suggest that two aspects of female body shape-WHR and BMIare differentially associated with judgments of dynamic and static attractiveness. For overall attractiveness, both of these factors were important, as they were for dynamic attractiveness. For static attractiveness, however, WHR was more important than BMI. These results are somewhat different from those suggesting that BMI is most important in photographs (Tovée et al., 1999). One explanation might be that previous studies, for example Tovée et al. (1999), used a sample of women with a very wide range of BMI and WHR, and greater importance of BMI may have been due to the relatively higher variation in it than in WHR (although study 2 restricted the range of BMI and found comparable results). We did not attempt to control the range of body shapes in our study: we just recruited an opportunity sample of women. It is therefore possible that the results would change depending on the relative variation of BMI and WHR - the wider the variation the more important that factor would be. Nevertheless, it would be interesting to recruit extreme ranges of body shapes and see how this affected the results. Our sample was also homogenous in terms of age and ethnicity. Preferred body shapes do vary across raters of different ethnicity (e.g. Thompson, Sargent \& Kemper, 1996) and ecology (e.g. Tovée et al., 2006). In resource poor communities, for example, a thin body may indicate malnutrition, illness, or low social status and not be attractive as fatter bodies. Therefore, it would be interesting to see if the role of movement also varies across these cultural factors.

Although the aim of using motion-capture is to isolate motion cues from static cues, this process is not perfect. Some aspects of body shape might be perceptible from the walkers used (e.g., relative height, length of limbs, hip width). Therefore some aspects 
of body shape may have influenced the dynamic attractiveness ratings. However, the motion-capture process eliminated obvious cues including BMI and WHR, and the available static information was probably minimal. The fact that WHR and BMI seemed to operate differently across pictures and point-light walkers suggest that these two conditions convey different information. Another limitation is the fact that we used a fixed frontal perspective. Different visual perspectives provide different information and can lead to different aspects of body shape being attractive (e.g. Rilling et al., 2009; Marlowe, Apicella \& Reed, 2005). Future studies could offer multiple perspectives to provide fuller 3-dimensional information to raters. Viewpoint can affect recognition of identity from biological motion (Kuhlmann, de Lussanet, Lape, 2009), although in this case the frontal view was easiest. Whether viewpoint makes a difference on dynamic judgements on attractiveness is an interesting avenue for research.

Our findings lead us to question why people should pay heed to dynamic cues when assessing attractiveness. At first glance, it seems that dynamic cues could be faked, and fakeable cues should not be important (Krebs \& Dawkins, 1984). If all it takes to be attractive is to walk a certain way, we might expect people to do it easily and naturally, and consequently there should be little variation in that trait and it should not be attractive. However, body shape may indicate and/or constrain the way a woman can move, and so she cannot fake attractive motion because her body shape does not allow it. Our results suggest that different aspects of body shape might be conveyed differentially through static and dynamic cues. WHR seems to be more conveyed through static than dynamic cues, and BMI more through dynamic than static cues. However, it remains to be tested whether the attractiveness of gait can be faked, for example by asking people to walk attractively. If static cues do constrain dynamic cues, then people should not be able to alter the attractiveness of their walks much, and it should remain constrained by their 
body shape. On the other hand, if movement can change attractiveness, we might expect people to do so in situations when they want to attract a potential mate (or indeed decrease it when they do not wish to do so).

Another reason to pay attention to dynamic cues is that they may offer additional information over static cues relevant to mate value. We already know that gait can indicate age (Montepare \& Zebrowitz-McArthur, 1988), and this may be important when someone is too far away for static cues to indicate their age. Gait may also indicate whether somebody is in good condition in obvious ways such as whether they have a limp, but also possibly in more subtle ways. It would be interesting to test this possibility by isolating the gait of people when they are tired or ill and seeing if this affects judgments of attractiveness.

Our findings suggest that how a woman moves is at least as important as how she looks when judging her attractiveness. We hope that future studies of human physical attractiveness will consider movement for three reasons: first, to increase ecological validity over static images; second, to explore the unique effects of movement on attractiveness; and third, to explore whether movement can be changed to increase or decrease attractiveness in situations when doing so would be an advantage.

\section{References}

Ambady, N., Hallahan, M., \& Conner, B. (1999). Accuracy of judgments of sexual orientation from thin slices of behavior. Journal of Personality and Social Psychology, 77, 538-547. 
Barclay, C.D., Cutting, J. E., \& Kozlowski, L.T. (1978). Temporal and spatial factors in gait perception that influence gender recognition. Perception \& Psychophysics, 23, 145-152.

Bloj, M.G., Kersten, D., \& Hurlbert, A.C. (1999) Perception of three-dimensional shape influences colour perception through mutual illumination. Nature, 402, 877-879.

Cornelissen P.L., Tovée M.J., \& Bateson M. (2009). Patterns of subcutaneous fat deposition and the relationship between body mass index and waist-to-hip ratio: Implications for models of physical attractiveness. Journal of Theoretical Biology, 256, 343-350. doi: 10.1016/j.jtbi.2008.09.041

Cutting, J.E., \& Kozlowski, L.T. (1977). Recognizing friends by their walk: Gait perception without familiarity cues. Bulletin of the Psychonomic Society, 9, 353356.

Dittrich, W.H. (1993). Action categories and the perception of biological motion. Perception, 22, 15-22.

Fink, B., Seydel, H., Manning, J.T., \& Kappeler, P.M. (2007). A preliminary investigation of the associations between digit ratio and women's perception of men’s dance. Personality and Individual Differences, 42, 381-390.

Fink, B., Weege, B., Neave, N., Pham, M. N., \& Shackelford, T. K. (2015). Integrating body movement into attractiveness research. Frontiers in Psychology, 6, 220.

Johansson, G. (1973). Visual perception of biological motion and a model for its analysis. Perception \& Psychophysics, 1, 201-211.

Knappmeyer, B., Thornton, I. M., Etcoff, N., \& Bülthoff, H. H. (2002). Facial motion and the perception of facial attractiveness. Journal of Vision, 2, 612-612. 
Kramer, R. S., Arend, I., \& Ward, R. (2010). Perceived health from biological motion predicts voting behaviour. Quarterly Journal of Experimental Psychology, 63(4), 625-632.

Krebs, J. R., \& Dawkins, R. (1984). Animal signals: mind-reading and manipulation. Behavioural ecology: an evolutionary approach, 2nd edn (ed. JR Krebs \& NB Davies), 380-402.

Kuhlmann, S., de Lussanet, M. H., \& Lappe, M. (2009). Perception of limited-lifetime biological motion from different viewpoints. Journal of Vision, 9(10), 11-11.

Lassek, W. \& Gaulin S. (2007). Waist-hip ratio and cognitive ability: is gluteofemoral fat a privileged store of neurodevelopmental resources? Evolution and Human Behavior, 29, 26-34.

Marlowe, F., Apicella, C., \& Reed, D. (2005). Men's preferences for women's profile waist-to-hip ratio in two societies. Evolution and Human Behavior, 26(6), 458468.

Maynard Smith, J. (1956) Fertility, mating behaviour and sexual selection in Drosophila subobscura. Journal of Genetics, 54, 261-279.

Montepare, J.M., \& Zebrowitz-McArthur, L. (1988). Impressions of people created by age-related qualities of their gaits. Journal of Personality and Social Psychology, 55, 547-556.

Morrison, E.R., Gralewski, L., Campbell, N., \& Penton-Voak, I.S. (2007). Facial movement varies by sex and is related to attractiveness. Evolution and Human Behaviour, 28, 186-192.

Morrison, E., Clark, A.P., Gralewski, L., Campbell, N. \& Penton-Voak, I.S. (2009). Women's probability of conception is associated with their preference for 
flirtatious but not masculine facial movement. Archives of Sexual Behavior, 39, 1297-1304.

Provost, M.P., Troje, N.F., \& Quinsey, V.L. (2008). Short-term mating strategies and attraction to masculinity in point-light walkers. Evolution and Human Behavior, 29, 65-69.

Rilling, J. K., Kaufman, T. L., Smith, E. O., Patel, R., \& Worthman, C. M. (2009). Abdominal depth and waist circumference as influential determinants of human female attractiveness. Evolution and Human Behavior, 30(1), 21-31.

Rowland, W.J. (1995). Do female sticklebacks care about male courtship vigour? Manipulation of display tempo using video playback. Behavior, 132, 951-961.

Singh, D. (1993). Adaptive significance of female physical attractiveness: Role of waitto hip ratio. Journal of Personality and Social Psychology, 65, 293-307.

Singer, F., Riechert, S.E., Xu, H., Morris, A.W., Becker, E., Hale, J.A., Noureddine, M.A. (2000) Analysis of courtship success in the funnel-web spider Agenenopsis aperta. Behaviour, 137, 93-117.

Thompson, S. H., Sargent, R. G., \& Kemper, K. A. (1996). Black and white adolescent males' perceptions of ideal body size. Sex Roles, 34(5-6), 391-406.

Thoresen J. C., Vuong Q. C., Atkinson A. P. (2012). First impressions: gait cues drive reliable trait judgements. Cognition, 124, 261-271.

Tovée M.J., Maisey D.S., Emery J.L., Cornelissen P.L. (1999) Visual cues to female physical attractiveness. Proceedings of the Royal Society London B, 266, 211218.

Tovée, M. J., Swami, V., Furnham, A., \& Mangalparsad, R. (2006). Changing perceptions of attractiveness as observers are exposed to a different culture. Evolution and Human Behavior, 27(6), 443-456. 
Ullman, S. (1979). The interpretation of structure from motion. Proceedings of the Royal Society London B, 203(1153), 405-426.

Wass, P., Waldenström, U., Rössner, S., \& Hellberg, D. (1997). An android body fat distribution in females impairs the pregnancy rate of in-vitro fertilization-embryo transfer. Human Reproduction, 12, 2057-2060.

Zaadstra, B.M., Seidell, J.C., Noord, P.A.H.V., Velde, E.R.T., Habbema, J.D.F., Vrieskwijk, B., \& Karbaat, J., (1993). Fat and female fecundity: prospective study of effect of body fat distribution on conception rates. British Medical Journal, 306, 484-487. 


\begin{tabular}{lcccc} 
& Mean & SD & Minimum & Maximum \\
& & & & \\
\hline WHR & 0.77 & 0.05 & 0.64 & 0.87 \\
BMI $\left(\mathrm{Kgm}^{-2}\right)$ & 22.78 & 2.88 & 17.48 & 30.60 \\
Attractiveness (1-7) & & & & \\
$\quad$ Static & 3.96 & 0.62 & 3.00 & 5.32 \\
Static+dynamic & 3.80 & 0.64 & 2.40 & 5.28 \\
Dynamic & 3.74 & 0.61 & 2.58 & 4.82 \\
\hline
\end{tabular}

Table 1: Descriptive statistics. None of the variables was significantly different from a normal distribution using Kolmogorov-Smirnov test. 


\begin{tabular}{lll} 
Predictor & $\beta$ & $\mathrm{T}(\mathrm{df}=36)$ \\
\hline Static attractiveness & $0.39 * *$ & 3.01 \\
Dynamic attractiveness & $0.58^{* *}$ & 4.49 \\
${ }^{* *} \mathrm{p}<0.01$ & &
\end{tabular}

Table 2: Regression model predicting static+dynamic attractiveness from dynamic and static cues. 


\begin{tabular}{|c|c|c|c|}
\hline Dependent variable & Predictor & $\beta$ & $\mathrm{t}$ \\
\hline \multirow[t]{2}{*}{ Static attractiveness } & BMI $_{\text {dev_static }}$ & -0.24 & -1.40 \\
\hline & WHR & $-0.51 * *$ & -3.08 \\
\hline \multirow[t]{2}{*}{ Dynamic attractiveness } & BMI & $-0.52 * *$ & -3.80 \\
\hline & WHR & $-0.29 *$ & -2.10 \\
\hline \multirow[t]{2}{*}{ Static+dynamic attractiveness } & BMIdev_static_dynamic & $-0.41^{* *}$ & -2.92 \\
\hline & WHR & $-0.46^{* *}$ & -3.37 \\
\hline
\end{tabular}

${ }^{*} \mathrm{p}<.05 .{ }^{* *} \mathrm{p}<.01$.

Table 3: Regression models predicting attractiveness from BMI and WHR. 


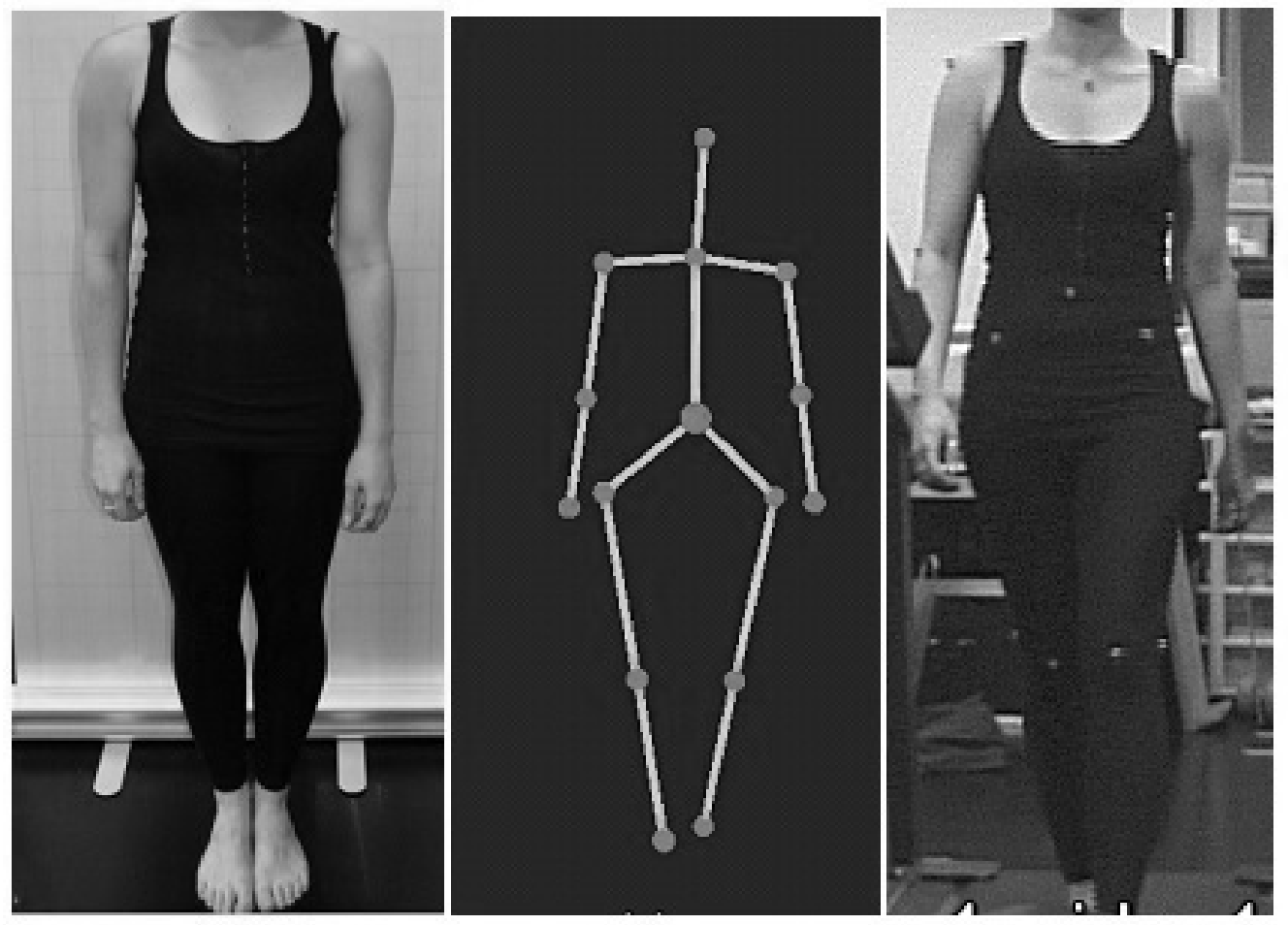

Figure 1: The stimuli presented to the raters. Photograph (left), point-light walker (centre) and still frame from video (right). Stimuli were presented in colour. 

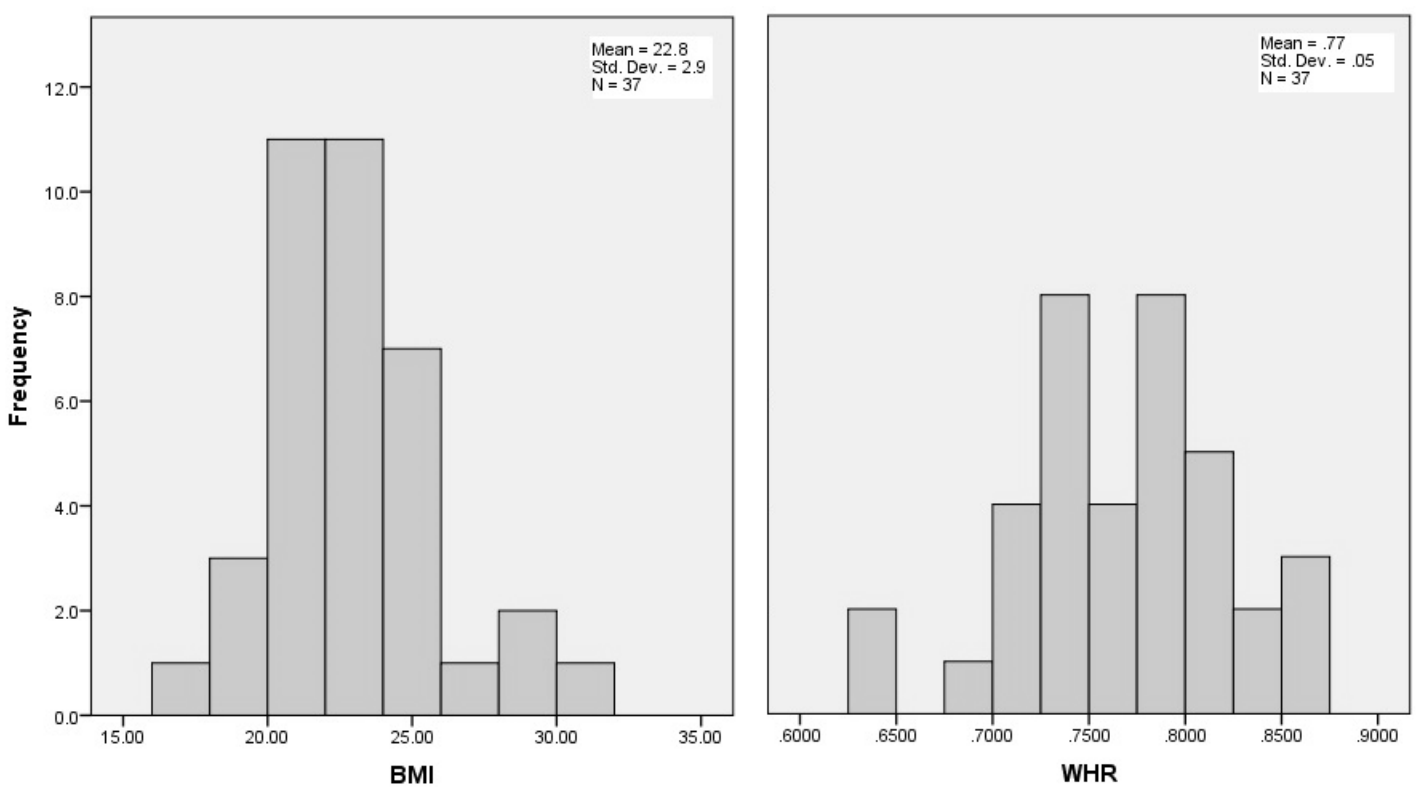

Figure 2: Histograms showing the distributions of BMI and WHR in the sample of models. 